\section{APORTES TEÓRICO-METODOLÓGICOS \\ PARA UN ESTUDIO COMPLEJO \\ DE LA REPRESENTACIÓN POLÍTICA \\ DE LAS MUJERES}

THEORETICAL-METHODOLOGICAL

CONTRIBUTIONS FOR A COMPLEX STUDY OF

THE POLITICAL REPRESENTATION OF WOMEN

\section{PAULA BEDÍN ·}

Becaria posdoctoral del CONICET con sede en el Centro de Estudios Históricos del Departamento de Historia de la Facultad de Humanidades, Universidad Nacional de Mar Del Plata (Argentina).

E-mail: paulabedinunmdp@gmail.com

\section{Resumen}

Los estudios que analizan la representación política de las mujeres han utilizado las afirmaciones de la teoría de la masa crítica como marco metodológico para el análisis empírico. No obstante, recientemente han surgido nuevos posicionamientos teóricos que se presentan como antagónicos a la teoría de la masa crítica. Estas perspectivas prestan mayor atención a las dimensiones sustantivas y simbólicas al proponer un análisis enfocado en cómo se construyen discursivamente. En el presente artículo sostendremos que es necesario producir una articulación entre ambos paradigmas para entender en toda su complejidad la representación política de las mujeres.

\section{Registro bibliográfico}

BEDÍN, PAULA «Aportes teórico-metodológicos para un estudio complejo de la representación política de las mujeres», en: ESTUDIOS SOCIALES, revista universitaria semestral, año XXIX, $n^{\circ}$ 57, Santa Fe, Argentina, Universidad Nacional del Litoral, julio-diciembre, 2019, pp. 11-29.

\section{Abstract}

The studies on women's political representation use the Critical Mass Theory as a methodological framework for empirical analysis. Nevertheless, new theoretical positions are opposed to the Critical Mass Theory. These positions pay more attention to substantive and symbolic representation by proposing a kind of analysis which is focused on how women's political representation is discursively constructed. In the present paper we claim that some sort of articulation between them is needed in order to fully understand the complexity of women's political representation.

\section{Descriptores · Describers}

Representación política de las Mujeres / Teoría de la Masa Crítica / Declaraciones Representativas Women's Political Representation / Critical Mass Theory / Representative Claims

Recibido: 22 / 05 / 2018 Aprobado: 02 / 04 / 2019 


\section{INTRODUCCIÓN}

Por lo general, en las investigaciones que analizan la representación política de las mujeres se han reconocido tres dimensiones: la descriptiva, la sustantiva y la simbólica. Dichas dimensiones fueron elaboradas por Pitkin en su clásico libro The concept of representation (1967). Según la autora, desde una concepción descriptiva de la representación se considera que el representante «está por» quienes comparten características similares a él y, por ello, representa los intereses de aquellos que poseen sus mismas características. Una buena representación descriptiva se logra cuando las legislaturas son un reflejo o espejo exacto de la nación, de la opinión pública o del electorado. En cambio, la dimensión sustantiva presta especial atención a lo que los representantes hacen. Su función específica consiste en un ejercicio independiente del juicio por parte de los representantes, pero conociendo anticipadamente los intereses y necesidades de los representados.

La dimensión simbólica es la menos desarrollada por Pitkin puesto que es asociada a ciertas estrategias de manipulación de los líderes políticos. Según la autora, esta dimensión tiene lugar cuando una cosa o persona se hace presente a través de símbolos. Un retazo de tela con algunos colores y/o dibujos o una canción, como la bandera o el himno nacional, pueden representar a una nación entera. En este sentido, los líderes crean símbolos no para generar un proceso de persuasión racional sino más bien para generar reacciones afectivas y hábitos. Aquí la representación ya no es un actuar por otros u ofrecer información sobre los representados sino una actividad ligada con convencer al pueblo de que acepte a un político como su representante simbólico.

La dimensión descriptiva y la sustantiva fueron las más elaboradas por los primeros estudios sobre la representación política de las mujeres en tanto han fundado sus investigaciones en las afirmaciones de la teoría de la masa crítica como marco metodológico para el análisis empírico. La principal tesis de esta teoría es que es necesario un porcentaje igual o mayor al $30 \%$ de representantes mujeres para provocar un cambio de política en favor de los intereses y los derechos de las mujeres. Quienes han adoptado esta teoría han privilegiado la dimensión descriptiva, investigando cómo una ampliación en dicha dimensión provoca efectos positivos también en las dimensiones sustantivas y simbólicas.

En contraposición a esta teoría, han surgido recientemente nuevos posicionamientos teóricos que se presentan como antagónicos a la teoría de la masa crítica. Estas perspectivas, que se caracterizan como más dinámicas, le señalan ciertas 
carencias teóricas y metodológicas. Asimismo, centran su atención en especial en las dimensiones sustantivas y simbólicas anteriormente poco tematizadas o subordinadas a la descriptiva. Incluso, como veremos en el transcurso del artículo, algunas teóricas argumentan que es la dimensión simbólica, y no la descriptiva, la que configura las otras dos dimensiones.

Aunque este paradigma «dinámico» se distancie críticamente del anterior, en el presente artículo afirmaremos que es necesario producir una articulación entre ellos para realizar un estudio complejo de la representación política de las mujeres teniendo en cuenta en profundidad todas sus dimensiones. Para mostrar la necesidad de dicha integración, en primer lugar, explicaremos en qué consiste la teoría de la masa crítica y qué tipo de estudios empíricos se han sustentado en ella. En segundo lugar, señalaremos las críticas que ha recibido por parte de quienes afirman postular una teoría más dinámica de la representación política. En tercer lugar, presentaremos brevemente en qué consisten estas perspectivas dinámicas para luego caracterizar los estudios empíricos que utilizaron esta teoría como base metodológica. Por último, realizaremos un breve resumen de los aportes de ambos enfoques en tanto volveremos a referirnos a los aspectos de la representación política de las mujeres que cada una de ellas ilumina. En ese sentido, este artículo no solo tiene como objetivo explicar y mostrar la necesidad de integración de ambos paradigmas sino también brindar un estado de la cuestión de los estudios empíricos inscriptos en cada uno de ellos.

\section{LA TEORÍA DE LA MASA CRÍTICA. LA CENTRALIDAD DE LA DIMENSIÓN DESCRIPTIVA Y SU IMPACTO EN LA SUSTANTIVA}

El inicio de este debate puede situarse en la publicación de un artículo, ahora clásico, que tenía como finalidad brindar un marco teórico para comprender el concepto de «masa crítica». Este texto, llamado «De una pequeña a una gran minoría: las mujeres en la política escandinava», de Drude Dahlerup (1988), presentaba el concepto de «masa crítica» como modo de caracterizar algunos estudios previos cuya temática era la inclusión de grupos minoritarios de mujeres a áreas tradicionalmente dominadas por hombres. Uno de esos estudios previos fue «Some Effects of Proportions on Group Life: Skewed Sex Ratios and Responses to Token Women» (KANTER, 1977). En este artículo la preocupación central de Kanter era mostrar su estatus simbólico en espacios tradicionalmente ocupados por varones. 
Dicho estatus genera estereotipos y un doble estándar al que se somete a las mujeres. Al no considerarse sus obligaciones familiares, nunca obtienen una licencia por maternidad. Sus salarios son más bajos y difícilmente asciendan. No logran poseer una autoridad legítima y carecen de aliados. Sufren hostigamiento sexual y tensiones psíquicas. Todo ello las conduce a una baja eficiencia y a una alta tasa de deserción (DAHLERUP, I988: 279). La única forma de contrarrestar ese «simbolismo», para Kanter, es lograr incrementar el número de mujeres presentes en dichos espacios hasta llegar a ocupar un $35 \%$ de los puestos. Únicamente en ese porcentaje los miembros de la minoría logran salir del estatus simbólico para ser potencialmente aliados y afectar la cultura del grupo. Incluso pueden ser identificados como individuos diferenciados entre sí y de la mayoría.

El impacto de esta investigación fue muy grande puesto que el $30 \%$ es el porcentaje mínimo solicitado y adoptado en la mayoría de leyes de cuotas alrededor del mundo. De igual modo, abrió el debate sobre si la cantidad menor o mayor de personas pertenecientes a minorías es un factor determinante tanto para salir del estatus simbólico como para lograr hacer la diferencia (NORRIS, I996; YODER, I99I). Esta pregunta es justamente la que intenta responder Dahlerup, quien caracterizó como "masa crítica» al porcentaje que Kanter marcaba como necesario para la salida del simbolismo. La masa crítica, en términos físicos, es un proceso que tiene lugar en una entidad o espacio aislado en el cual se necesita una masa crítica para que tenga lugar una reacción en cadena. Aplicado al estudio de la representación de las mujeres, este concepto refiere a la pregunta de cuántas mujeres se requieren para producir una diferencia en la política.

No obstante, Dahlerup toma distancia de la teoría de la masa crítica al plantear una serie de objeciones a sus presupuestos. En primer lugar, afirma que el «simbolismo» no se debe a la escasa presencia en las corporaciones sino al hecho de que son mujeres. El estatus de minoría dentro de la empresa está relacionado con el estatus de minoría que sufren en la sociedad en general. Esto provoca que, incluso cuando son mayoría, no produzcan necesariamente transformaciones. Esto último quedó demostrado en aquellas fábricas donde las mujeres eran la amplia mayoría y no pudieron mejorar sus salarios ni las condiciones de trabajo (DAHLERUP, 1988: 278).

En segundo lugar, argumenta que no hay una relación necesaria y directa entre el número de mujeres representantes y las diferencias que provocan. El problema es que cuando esos cambios tienen lugar es imposible aislar los efectos del incre- 
mento de la proporción respecto de lo que sucede afuera de los parlamentos. Solo cuando se produce un cambio en el clima social, en las instituciones políticas, se puede esperar una mayor incidencia de las minorías. Sin embargo, este cambio del contexto social puede reflejarse en los parlamentos «cuando la primera mujer entra en la sala de reunión» (DAHLERUP, 2006: 513).

Tampoco existen acuerdos unánimes en cuanto a qué significa «hacer la diferencia». Algunos estudios consideran que se produce una diferencia en los «asuntos de mujeres», otros en los "asuntos feministas», y otros en "cuestiones de género» (BECKWITH, COWELL-MEYERS, 2007). Asimismo, esta idea posee ciertos problemas metodológicos que Dahlerup denomina como «falacia de la diferencia». Gran parte de los estudios empíricos posee suficientes pruebas para identificar algunas diferencias en política entre los varones y las mujeres, como, por ejemplo, que estas últimas tienden a ser más activas cuando se trata de introducir políticas de igualdad en la agenda. El problema es que este tipo de estudios subestima su capacidad de influencia hacia sus colegas varones para cambiar dicha agenda.

Por estos motivos, la dificultad de la teoría de la masa crítica tiene que ver con postular como fundamental el incremento numérico de representantes como condición de posibilidad de las diferencias en las políticas que puedan provocar, sin tener en cuenta factores como el contexto político, el ambiente social y político, las maquinarias feministas estatales, los discursos prevalecientes, la construcción de coaliciones y la fuerza del movimiento feminista, entre otros. Es por ello que los estudios que intentan comprobar empíricamente si hubo o no algún tipo de impacto en la dimensión sustantiva arrojan resultados disímiles. Tanto es así que no solo la controversia aparece cuando son aplicados en distintos contextos geográficos, culturales y políticos sino también cuando son aplicados al mismo caso. ${ }^{1}$

La discrepancia entre los resultados es tan profunda que termina por dividir en categorías opuestas las conclusiones. Están aquellas que tienen una posición pesimista (GREY, 2002; STUDLAR Y MCALLISTER, 2002) en contraposición con los optimistas (JAQUETTE, I997; STAUdT, 1996; CARROLL, I995). Tanto las optimistas como las pesimistas no solo toman como única variable a ser tenida en cuenta el número, sino que confunden la representación sustantiva como proceso y la representación sustantiva como resultado. Las pesimistas se enfocan en los resultados, con frecuencia escasos, para afirmar que una mayor presencia no cambia

1] Para el caso de Argentina (HTUN Y JONES, 2002; FRANCESCHET Y PISCOPO, 2008). 
dramáticamente las políticas públicas. Las visiones optimistas se concentran en la cantidad de proyectos presentados y el patrocinio conjunto entre legisladoras a favor de los derechos de las mujeres (FRANCESCHET Y PISCOPO, 2008).

Quienes son optimistas respecto del vínculo entre representación descriptiva y sustantiva en sus dos acepciones desoyen la objeción que Dahlerup hacía a la masa crítica en torno a la imposibilidad de recortar tanto los porcentajes como los logros de las representantes de un contexto más amplio. La aplicación de la teoría de la masa crítica a todo contexto la vuelve una teoría con pretensiones universalistas que deja sin tematizar las condiciones contextuales que podrían incidir en la representación sustantiva (BECKWITH y COWELL-MEYERS, 2007). En ese sentido, algunos estudios demuestran que en lugares donde el incremento de la representación no fue alto se obtuvieron políticas de género progresistas gracias a la presión que los movimientos de mujeres ejercían (SACCHET, 2008: 37I).

De este modo, la relación entre dimensión descriptiva y sustantiva debería ser concebida no ya como necesaria (DODson, 2006) sino como débil, complicada y contingente (MACKAY, 2008). Teniendo en cuenta esto último, algunas teóricas de la masa crítica han moderado las aspiraciones universalistas de esta perspectiva. Uno de ellos fue el trabajo realizado por Thomas (THOMAs, I991, 1994). Esta autora, luego de analizar los diferentes efectos de un mayor número de mujeres en I2 legislaturas en Estados Unidos, identificó algunos cambios en las prioridades políticas y en los resultados, especialmente en el patrocinio y la aprobación de proyectos asociados a asuntos de mujeres. Sin embargo, no encontró diferencias provocadas en el proceso legislativo, aunque advierte que esto puede ser distinto en otros contextos (THOMAS, 1994: 154).

Más allá de los desacuerdos entre las teóricas, desde la perspectiva de la masa crítica se provee una forma de entender la centralidad de la dimensión descriptiva que permite realizar ciertos estudios empíricos de gran relevancia. Por ejemplo, en Argentina se han analizado la cantidad de mujeres que ejercía como representante antes y después de la implementación del cupo o de la paridad (ARCHENTI, 2OI4; ARCHENTI Y TULA, 2008, 20I4; CAMINOTTI, 2OI7) y el impacto de ello en la cantidad y las temáticas de los proyectos legislativos que presentaron (FRANCESCHET Y PISCOPO 2008). Asimismo, se ha estudiado el perfil de las representantes (MARX, BORNE y CAMINOTTI, 2007) y su distribución en las comisiones. Este último estudio es fundamental porque muestra que no basta con incrementar el número de representantes para terminar con las desigualdades en tanto existe una 
división sexual del trabajo legislativo que las excluye de las comisiones poderosas ${ }^{2}$ (PIATTI-CROCKER, 2OI4; BORNER, CAMINOTTI, MARX Y RODRÍGUEZ GUSTÁ, 2009; PÉREZ, 2OI4; BARNES, 2OI3 Y 2OI4).

En este sentido, una vez que se ha moderado la universalidad y el determinismo entre una mayor presencia y un cambio en la política, los resultados que arrojan los estudios que analizan los impactos de un incremento en la dimensión descriptiva de la representación política de las mujeres son fundamentales para comprender su complejidad. Sin estos estudios perderíamos de vista cuáles son los cambios concretos que ha generado en la política un mayor ingreso y cuáles son los desafíos que todavía operan excluyendo a las mujeres de los espacios de poder.

\section{EL ANÁLISIS DE LA DIMENSIÓN SIMBÓLICA DESDE EL MARCO TEÓRICO DE LA MASA CRÍTICA}

Algunas investigaciones en torno a las cuotas de género argumentan a favor de su implementación en tanto consideran que un incremento de mujeres en política genera «modelos a seguir» (KARP Y BANDUCCI, 2008). Dichos modelos provocarían un impacto simbólico que promueve en las mujeres en general un mayor interés hacia la política y su empoderamiento. No obstante, no todas las autoras están de acuerdo en que una modificación en la dimensión descriptiva provoca necesariamente una transformación en la dimensión simbólica.

Quienes son optimistas afirman que el aumento de la presencia de las representantes en los parlamentos moldea nuestra percepción colectiva sobre cómo los/as líderes políticos/as se ven y qué lugares logran ocupar. Sostienen que estos cambios transforman las expectativas de las más jóvenes y las alientan a ingresar a los partidos políticos y postularse para cargos electivos (KITTILsON, 2005: 643). A partir de esta afirmación, algunas investigaciones se enfocaron en cómo estos modelos a seguir femeninos transformaron la percepción de las mujeres respecto de la confianza en las legislaturas (SCHWINDT-BAYER Y MISHLER, 2005) y las responsabilidades de los gobiernos hacia los ciudadanos y ciudadanas (ATKESON Y CARILLO, 2007). Afirmaron que la presencia de candidatas competitivas estimula

2] Las comisiones más poderosas en el caso de Argentina son Asuntos Constitucionales, Presupuesto y Hacienda y Relaciones exteriores y culto (CALVO, 2014). 
en las ciudadanas debates e intentos de participación política (WOLBRECHT Y CAMPBELL, 2007; ATKESON, 2003). Además, el efecto simbólico entre una mayor presencia en los parlamentos y la confianza de las ciudadanas en las habilidades de sus congéneres para gobernar produce un círculo virtuoso que refuerza un cambio en el empoderamiento de las mujeres como líderes políticos (ALEXANDER, 2OI2).

Bajo esta óptica, se considera entonces que el potencial del cupo femenino se extiende más allá del incremento del número. Esta puede ser una medida formal que pone en marcha cambios a nivel social al enviar un mensaje a los agentes institucionales y sociales sobre las desigualdades de género (SACCHET, 2008: 377). En este sentido, el gran aporte de los cupos reside en los impactos subjetivos fundamentales para el cambio político en tanto contribuye al estrechamiento de la grieta de la desigualdad de género (DESPOSATO Y NORRANDER, 2009).

A diferencia de estas posiciones, encontramos estudios de caso que presentan enfoques opuestos, o que moderan esta visión optimista, sobre el vínculo entre representación descriptiva y simbólica. En ellos se sostiene que, si bien es más probable que las mujeres provoquen una evaluación favorable sobre el gobierno cuando ocupan más cantidad de puestos de poder, no existe evidencia empírica de que esta percepción se traduzca en actitudes y comportamientos políticos en general (LAWLESS, 2004). Incluso un incremento rápido del número de mujeres en la política ha provocado un «efecto etiqueta» sobre las legisladoras. Al ser construidas simbólicamente como «las mujeres del cupo», intentan deshacerse de dicho estigma disminuyendo su voluntad de «actuar por» las mujeres y de realizar alianzas con otras legisladoras de distintos partidos políticos (FRANCESCHET Y PISCOPO, 2008: 4OI).

Por su parte, Zetterberg señala que las posiciones que afirman un vínculo determinante de la representación descriptiva sobre el empoderamiento e involucramiento político tienden a generalizar los efectos y olvidar otros factores (zETTERBERG, 2009). Por ejemplo, se podría afirmar que un incremento del número, gracias a leyes de cupo femenino o paridad, tiene lugar en los países donde ya existe un empoderamiento como condición de posibilidad de la ley.

Las refutaciones a las afirmaciones de la «masa crítica» también provinieron de teorías que brindan una concepción más dinámica de la representación política. Como veremos en el siguiente apartado, estas proponen dejar de priorizar la dimensión descriptiva como primordial para una mejor representación sustantiva y simbólica. 


\section{DE LA MASA CRÍTICA A LOS ACTORES CRÍTICOS}

Una serie de autoras han visto la necesidad de dejar de lado la teoría de la masa crítica para comenzar a pensar la representación sustantiva y simbólica de las desde un marco teórico más «dinámico». Para dichas autoras, la teoría de las declaraciones representativas de Michel Saward fue crucial para lograr este objetivo. Dicho autor le otorga una importancia fundamental a la dimensión simbólica de la representación política al concebirla como un proceso dinámico y performativo (BUTLER, 1999 Y 2009) realizado por diversos actores sociales críticos que configuran parcialmente las identidades o audiencias que afirman representar (SAWARD, 2OIO). Desde este enfoque, la representación política es vista como un proceso llevado adelante por actores o productores de declaraciones representativas gracias a las cuales, al ofrecer un retrato o descripción de sí mismos, pretenden moldear a su electorado o audiencia (SAWARD, 2OI2).

La aplicación de esta teoría al análisis de la representación política de las mujeres generó una serie de refutaciones hacia la teoría de la masa crítica. En primer lugar, se rechaza la idea que da por supuesto que estas conforman un grupo, ignorando o invisibilizando las diferencias entre ellas. En segundo lugar, que son las únicas que llevan adelante sus propios intereses. Por último, sostienen que examinar la representación sustantiva (en adelante RSM) como aquello que tiene lugar únicamente cuando se introducen y aprueban políticas gracias a las representantes en los parlamentos, limita la representación a un conjunto de actores, a un solo sitio y a un modo de representación política (WELDON, 2002; LOVENDUSKI, 2005; CELIS, 2006).

Con este enfoque, la RSM ya no depende de un incremento de representantes políticas hasta formar una «masa crítica» sino más bien de actores críticos que puedan promover políticas que ayuden a mejorar la vida de las mujeres (DAHLERUP, I988). En este sentido, quienes encarnan la RSM son «actores críticos» diversos (CHILDS Y KROOK, 2006; CHILDS Y WITHEY, 2006). En algunas situaciones, varones individuales pueden jugar un rol crucial en el avance de las políticas de las mujeres (BALDEZ, 2OII; TAMERIUS, I995; CELIS, 2006). Asimismo, estos no son únicamente representantes políticos electos sino también un conjunto más amplio de jugadores posibles, como ministros, miembros de partidos políticos, burócratas, integrantes de ciertos grupos de la sociedad civil, referentes de movimientos sociales y ONG (WELDON, 2002). Más aún, esta teoría permitió analizar lugares por fuera de los parlamentos donde también se expresan los diversos actores críticos, como foros políticos, agencias, 
organizaciones no gubernamentales y de la sociedad civil (CHAPPELL, 2002; BANASZAK, BECKWITH Y DIETER, 2003; LOVENDUSKI, 2005; KANTOLA, 2006).

Desde estos estudios, no se presupone que un mayor número de mujeres produce una diferencia en la política en tanto reemplazan esa afirmación por la pregunta sobre cuáles son las condiciones que hacen que ciertos actores puedan obtener mayores logros. Una respuesta posible es que probablemente sean quienes ocupan lugares políticos de relevancia. Más aún si actúan en conjunto con otros actores críticos en distintos lugares, estableciendo alianzas estratégicas y buscando políticas que estén en sintonía con el partido que gobierna (BECKWITH Y COWELL-MEYERS, 2007; KANTOLA y OUTSHOORN, 2007). No obstante, se advierte la necesidad de explorar la competencia y el conflicto que pueden existir entre ellos en tanto posean distintas concepciones respecto de qué es la representación sustantiva con relación a su contenido, dirección o propósito (CELIS, CHILDS, KANTOLA Y KROOK, 2008: 104). Teniendo en cuenta esto último, Saward propone la idea de pensar los actores más bien como «declarantes críticos», puesto que esta concepción posee la ventaja de enfatizar el impacto de los actos de realización de declaraciones más que en el estatus de quienes las realizan (SAWARD, 20IO: I25).

Igualmente, desde esta perspectiva se cuestiona la idea tradicional sobre la RSM desde la que se sostiene que las mujeres deben representar los intereses de las mujeres. El principal problema que señalan es cómo determinar dichos intereses sin homogeneizar y esencializar sus identidades y demandas. En este punto, las autoras enfatizan sobre la importancia de advertir que las preocupaciones feministas son solo una faceta de la RSM. Incluso algunas consideran necesario distinguir entre RSM y la representación sustantiva de las feministas (MACKAY, 2008). En sintonía con esta idea, se ha señalado que la RSM contempla tanto los intereses de las feministas como de quienes no afirman ser feministas e incluso de las representantes conservadoras. Una buena RSM solo puede darse si se tienen en cuenta la diversidad y las ideologías en conflicto como parte fundamental para su configuración (CELIS, 2008 y 2009).

En este mismo sentido, Celis, Childs, Kantola y Krook (2014) han utilizado el marco teórico de los actores críticos para realizar un trabajo empírico sobre la configuración de la RSM en Bélgica, Finlandia e Inglaterra, teniendo en cuenta una multiplicidad de actores en contextos históricos y geográficos determinados. En dicho artículo, las autoras, en lugar de enfocarse únicamente en las representantes electas, reconocen como actores fundamentales también a los varones representantes y a integrantes de la sociedad civil que participan activamente por fuera de las legislaturas en 
la producción de declaraciones. Una vez que los actores han sido definidos, se busca determinar cuándo una declaración realmente está siendo realizada en nombre de las mujeres. Esto sucede cuando son construidas como de importancia para ellas en tanto las afecta directamente. De igual manera, cuando son discutidas en términos de diferencia de género o presentadas como productoras de efectos sobre el género.

El siguiente paso consiste en un análisis de las múltiples declaraciones representativas. Este implica tomar nota no solo de cómo varios actores retratan los intereses sino también qué concepción normativa está implicada en cada representación. Esto último involucra determinar, por ejemplo, si las declaraciones convocan ideales sobre «la» mujer que refuerzan las relaciones de género tradicionales, buscan promover la igualdad de género o reflejan una combinación de ambos. No obstante, este aspecto corresponde más a un estudio de la dimensión simbólica que de la sustantiva (LOMBARDo y MEIER, 20I7).

En América Latina existen pocos estudios de caso que hayan aplicado estas líneas teóricas en cuanto a la RSM. Encontramos el estudio realizado por Niki Johnson (20I4), quien analiza el éxito de la bancada femenina en Uruguay examinando el impacto favorable de las declaraciones de diversos actores críticos, como legisladoras y legisladores, miembros de distintos partidos políticos y organizaciones sociales y activistas. También menciona a actores como legisladores varones y medios de comunicación que tendieron a despreciar y socavar la bancada. Otro trabajo que se vale de esta perspectiva teórico-metodológica es el de Stoffel (stoffel, 2008). En este artículo la autora refuta la idea que afirma que la actividad de ciertas ONG feministas en Chile no forman parte de la representación política. A diferencia de esta posición, sostiene que pueden ser consideradas como parte importante puesto que sus declaraciones representativas son reconocidas por una gran cantidad de chilenas, gracias a lo cual poseen el acceso a recursos de negociación y realización de políticas.

En Argentina se aplica esta línea teórica para mostrar cómo, para la aprobación del Cupo Sindical y el Protocolo Facultativo de la Convención sobre la Eliminación de toda forma de Discriminación Contra la Mujer (CEDAw), fue necesaria la articulación entre diversos declarantes críticos sociales (BORNER, CAMINOTTI, MARX Y RODRÍGUEZ GUSTÁ, 2009). En el mismo sentido, otra investigación advierte el impacto que tuvieron las redes promovidas por el Sistema de Naciones Unidas (onU Mujeres, PNUD, UNFPA y, en menor medida, UNICEF) en la configuración de una agenda legislativa de género gracias a la cual se lleva adelante una representación sustantiva fundada en los derechos humanos de las mujeres (RODRIGUEZ GUSTÁ Y MADERA, 2OI4). 


\section{EL ANÁLISIS DE LA DIMENSIÓN SIMBÓLICA DESDE}

\section{LA PERSPECTIVA DE LAS DECLARACIONES REPRESENTATIVAS}

Más recientes han sido los estudios que se enfocan en la dimensión simbólica desde la perspectiva de las declaraciones representativas. Uno de los pocos libros dedicados al tema es el de Lombardo y Meier (LOMbardo y MeIer, 2OI4). Desde una aproximación discursiva de la representación simbólica, las autoras afirman que los/as declarantes crean y evocan en sus discursos concepciones sobre el género a través de las cuales producen performativamente las identidades y los intereses de quienes afirman representar. Al configurar su propia representación política a partir de una interpretación y evocación específica de las relaciones de género, de cierta concepción de qué es ser mujer y cuáles son sus intereses, no solo simbolizan dichas concepciones sino también las desestabilizan o refuerzan.

A este respecto, las autoras señalan que la representación simbólica posee un gran impacto en la representación descriptiva en tanto la primera brinda significado y valor a la segunda. Al proveer la descripción normativa de lo que es el género, la representación simbólica tiene el poder de «dar cuerpo» a la representación descriptiva. «Los estereotipos que son reproducidos en los discursos pueden deslegitimar o legitimar a las mujeres como representantes políticos. La representación simbólica del género es un factor decisivo en la aceptación de la representación descriptiva de las mujeres» (LOMBARdo y MEIER, 20I5: 136, la traducción es propia).

Así, un análisis de la representación descriptiva permite dar cuenta de cuáles son las normas que configuran las representaciones simbólicas y que, al mismo tiempo, funcionan como criterio regulador al establecer quiénes pueden llegar a ser representantes y quiénes no. Si la representación simbólica muestra a las mujeres como sujetos marginales de la esfera pública y a los varones como sujetos hegemónicos, confortablemente ubicados en el espacio público del trabajo y la política, se deduce quiénes obtendrán una mayor representación descriptiva.

En tanto, las autoras examinan cómo estas construcciones normativas del género impactan en la RSM. Muestran que las declaraciones no son únicamente un problema de quién está diciendo qué y en nombre de quién. Más bien es un problema de cómo actores representativos formulan sus declaraciones interactuando con un contexto más amplio de normas explícitas e implícitas que pueden confirmar o desestabilizar el contexto simbólico y normativo dominante (LOMBARDO Y MEIER, 2OI4: I48). 
Utilizando estas categorías de análisis, las autoras presentan algunas imágenes o fotografías. La más significativa de las analizadas es una foto de la ministra de Defensa espańola embarazada mientras supervisa por primera vez las tropas de las fuerzas armadas. En este caso, la fotografía ocasionó un gran revuelo en Espańa. En una primera lectura de la imagen, las autoras postulan que su impacto se debió a que se muestra a una mujer que está por ser madre en una posición de liderazgo. La particularidad de este liderazgo es que es ejercido por una mujer embarazada, símbolo de «feminidad», sobre la armada nacional, la cual simboliza la masculinidad exacerbada. Además, la ministra está ocupando un espacio y un liderazgo que tiende a ser asociado y ocupado por varones. La atracción de la foto también se debe a que la maternidad simboliza la vida y las milicias, más bien, la muerte. Esta presencia de la vida en un contexto que simboliza la muerte abrió el debate sobre el rol de las fuerzas armadas. La imagen invirtió las expectativas arraigadas sobre el lugar que ocupan la mujer y el varón y las jerarquías que han sido perpetradas a través de códigos culturales.

En una segunda lectura, las autoras reflexionan sobre cómo la foto construye cierta representación del género y propone diversas interpretaciones a quienes la reciben. Una de las interpretaciones abordadas es la de algunas feministas, para quienes esa imagen simboliza la llegada de las mujeres a espacios que antes eran exclusivamente masculinos. Según ellas, allí se muestra un nuevo rol de la armada que no solo incluye combates sino también operaciones humanitarias. Sin embargo, para ciertos actores políticos conservadores, la foto generó un fuerte escepticismo respecto de la capacidad que una ministra embarazada puede tener para manejar la defensa de un país. Enfatizaron en la posibilidad de que no pudiera llevar adelante sus responsabilidades luego de ser madre. Para los conservadores, la ministra embarazada que supervisa las tropas simboliza su incompetencia e incapacidad para lidiar con las tareas para las que fue designada.

A través de este relato, las autoras evidencian que la imagen es política y que lo que se discute a partir de ella son asuntos de representación política en general y de la representación simbólica en particular. Pero además, el debate nos muestra que hay diferentes interpretaciones sobre qué simboliza una imagen política. El sentido de un símbolo es disputado en el discurso político y posee diferentes significados para distintas personas. Los gestos, imágenes, discursos, etc., son parte de la performance mediante la cual los declarantes críticos (en este caso, una ministra) producen declaraciones representativas. Estas implican ciertas simbolizaciones 
que serán interpretadas, aceptadas, rechazadas y disputadas, de modos diversos, por quienes reciben esas declaraciones.

La imagen de la ministra es también un símbolo generizado. Sugiere, pero al mismo tiempo pone en jaque sentidos, creencias y roles que son asociados a las mujeres y a los varones. De este modo, la realización de declaraciones representativas es fundamental en la construcción de valores generizados en tanto algunas fortalecerán concepciones más tradicionales en torno al género pero otras producirán simbólicamente nuevos modos de representación. Lo que no se puede en ningún caso es negar la importancia que tiene esa producción de sentidos y símbolos de las declaraciones representativas en la construcción normativa del género.

En este sentido, esta nueva perspectiva sobre la representación política en su dimensión simbólica invierte la relación de determinación entre las dimensiones. Si anteriormente los estudios habían señalado el factor cuantitativo como determinante para la representación política de las mujeres, estas investigaciones darán cuenta de un factor cualitativo que pone el foco en cómo ciertas concepciones y estereotipos en el terreno de lo simbólico construyen un marco que regula el ingreso, los lugares y los roles que desempeñan tanto mujeres como varones. Así, la dimensión simbólica es la que configura las dimensiones descriptivas y sustantivas, y no lo contrario. Como veremos en el siguiente apartado, nuestra apuesta es mostrar ambas determinaciones como relevantes, imposibles de ser jerarquizadas, y cómo su articulación puede permitir elaborar una concepción compleja de la representación.

\section{POTENCIALIDADES DE LA ARTICULACIÓN DE AMBOS ENFOQUES}

A través del artículo, hemos explicado en qué consiste la teoría de la masa crítica y los debates que se generaron en torno a sus principales supuestos. No obstante, es necesario señalar que la adopción de esta perspectiva centrada en la dimensión descriptiva permite un análisis riguroso de: I) la cantidad de legisladoras luego de la implementación de acciones afirmativas o de la paridad de género; 2) la cantidad de mujeres en puestos ejecutivos e incluso dentro de la justicia o sindicatos; 3) la distribución en las comisiones legislativas y cuántas llegan a ocupar lugares destacados; 4) cuánto tiempo permanecen o no en estos espacios y por qué motivos 5) qué perfil poseen quienes llegan a ser representantes; y 6) cómo inciden todos estos datos en la representación sustantiva de las mujeres. 
En contraposición, presentamos aquellas concepciones que se denominan dinámicas. A partir de estas mostramos qué aspectos de la representación política habían quedado sin tematizar desde el punto de vista de la masa crítica y también cómo posibilitaron nuevos estudios sobre el tema. En este sentido, señalamos que la perspectiva dinámica ha posibilitado analizar: I) las articulaciones entre distintos actores críticos para que determinadas leyes en favor de las mujeres pudieran ser posibles; 2) la promoción de organismos internacionales y discursos en disputa respecto de coaliciones entre legisladoras de distintos partidos políticos; 3) la consideración de los varones como posibles declarantes a favor de una mejor RSM; 4) la presencia de las representantes conservadoras que se posicionan en contra de ciertos derechos e intereses de las mujeres; 5) la influencia en la RSM de instituciones nacionales e internacionales por fuera de las legislaturas tanto como de los movimientos sociales; 6) el modo en que los distintos declarantes críticos brindan interpretaciones en disputa sobre temáticas o imágenes relevantes a través de las cuales se refuerzan o se cuestionan normatividades en cuanto a las relaciones de género; y 7) cómo dichas construcciones discursivas configuran o moldean las dimensiones descriptivas y sustantivas.

No obstante, este último paradigma, al enfocarse en el análisis discursivo de la configuración de las tres dimensiones, desatiende los aspectos más cuantitativos de la representación política de las mujeres en su dimensión descriptiva. Nuestra afirmación con referencia a la necesidad de articular ambos puntos de vista responde a la convicción de que cada uno de ellos ilumina diferentes aspectos de la representación política que el otro desconoce. Por un lado, la teoría de la masa crítica ayudó a profundizar el conocimiento respecto de la dimensión descriptiva y su impacto en las dimensiones sustantivas y simbólicas. Por otro, las teorías más dinámicas prestaron más atención a la configuración discursiva de las dimensiones sustantiva y simbólicas en sí mismas. De igual manera, se enfocaron en la dimensión descriptiva pero para estudiar cómo esta puede estar regulada por la dimensión simbólica.

Como mostramos a través de los distintos apartados, ambos enfoques posibilitaron una diversidad de estudios empíricos que contribuyeron a captar en toda su complejidad la representación política de las mujeres. Teniendo en cuenta esto último, y a modo de conclusión, afirmamos que es necesario dejar de pensarlos como antagónicos en tanto su articulación puede posibilitar la realización de nuevos estudios que den como resultado una mayor complejidad en el análisis de la representación política de las mujeres. En otras palabras, dicha articulación nos 
invita a comprender que un mayor número de declarantes críticos puede provocar una modificación en las concepciones simbólicas tanto como un cambio simbólico en cuanto a los marcos normativos formales e informales puede provocar un mayor ingreso de declarantes críticos en posiciones relevantes de poder. Mostrar a través de estudios empíricos cómo tiene lugar esta relación mutua será la tarea principal de futuras investigaciones.

\section{Referencias bibliográficas}

ALEXANDER, AMY C. (2012): «Change in Women's Descriptive Representation and the Belief in Women's Ability to Govern: A Virtuous Cycle», en: Politics \& Gender, n 8, pp. 437-464.

ARCHENTI, NÉLIDA (2014): «El proceso hacia la paridad en América Latina. Argentina y Costa Rica», en: Revista Derecho Electoral, n 17, pp. 304-332.

ARCHENTI, NÉLIDA Y TULA, MARÍA INÉS (2008): «Algunas cuestiones iniciales sobre las leyes de cuotas», en: N. Archenti y M.I. Tula (eds.), Mujeres y política en América Latina. Sistemas electorales y cuotas de género, Buenos Aires, Heliasta.

ARCHENTI, NÉLIDA Y TULA, MARÍA INÉS (COORD.) (2014): La representación imperfecta. Logros y desafíos de las mujeres políticas, Buenos Aires, Eudeba. ATKESON, LONNA RAE (2003): Not All Cues Are Created Equal: The Conditional Impact of Female Candidates on Political Engagement, en: The Journal of Politics, vol. 65, n 4, pp. 1040-1061. ATKESON, LONNA RAE; CARRILLO, NANCY (2007): «More is Better: The Influence of Collective Female Descriptive Representation on External Efficacy», en: Politics \& Gender, n 3, pp. 79-101.
BANASZAK, LEE ANN; BECKWITH, KAREN; RUCHT, DIETER (2003): «When power relocates: Interactive changes in women's movements and states», en: L.A. Banaszak, K. Beckwith and D. Rucht (eds.), Women's Movements Facing the Reconfigured State, Cambridge, Cambridge University Press, pp. 1-29. BARNES, TIFFANY (2013): «Gender and Legislative Preferences: Evidence from the Argentine Provinces», en: Politics \& Gender, vol. 8, n 4, pp. 483-507.

BARNES, TIFFANY (2014): «Women's representation and legislative committee appointments: the case of the argentine Provinces», en: Revista Uruguaya de Ciencia Política, vol. 23, nº 2, pp. 135-161. BECKWITH, KAREN; COWELL-MEYERS, KIMBERLY (2007): «Sheer numbers: Critical representation thresholds and women's political representation", en: Perspectives on Politics, vol. $5, \mathrm{n}^{\circ} 3, \mathrm{pp}$. 553-565.

BORNER, JUTTA; CAMINOTTI, MARIANA; MARX, JUTTA; RODRÍGUEZ GUSTÁ, ANA LAURA (2009): Ideas, presencias y jerarquías políticas. Claro oscuros de la igualdad de género en el Congreso Nacional de Argentina, Buenos Aires, Prometeo. 
BUTLER, JUDITH (1998): «Actos performativos y constitución del género: un ensayo sobre fenomenología y teoría feminista», en: Debate Feminista, no 18, pp. 296-314.

BUTLER, JUDITH (1999): Gender Touble. Feminism and the subversion of Identity, New York, Routlegde.

BURNS, NANCY; LEHMAN SCHLOZMAN, KAY; VERBA, SIDNEY (2001): The Private Roots of Public Action: Gender, Equality and Political Participation, Cambridge, Harvard University Press.

CALVO, ERNESTO (2014): Legislator success in fragmented congresses in Argentina: Plurality cartels, minority presidents, and lawmaking, Cambridge, Cambridge University Press.

CAMINOTTI, MARIANA (2017): La paridad política en Argentina: avances y desafíos, Buenos Aires/ Lima/Panamá, Programa Naciones Unidas para el Desarrollo-PNUD/IDEA Internacional/Organización de las Naciones Unidas. Mujeres. Disponible en: http://www.ar.undp.org/content/argentina/es/ home/library/womens_empowerment/IPPArgentinaLibro/ (último ingreso: 14/02/2018).

CARROLL, SUSAN J. (ED.) (1995): Impact of Women in Public Office, New Brunswick, Rutgers University Press.

CELIS, KAREN (2006): «Substantive representation of women and the impact of descriptive representation. Case: The Belgian Lower House 1900-1979», en: Journal of Women, Politics and Policy, vol. 28, n² 2, pp. 85-114.

CELIS, KAREN (2008): «Studying women's substantive representation in legislatures: when representative acts, contexts and women's interests become important», en: Representation, vol. 44, no 2, pp. 111-123.

CELIS, KAREN (2009): «Substantive representation of women (and improving it): What it is and should be about?», en: Comparative European Politics, n 7 , pp. 95-113.
CELIS, KAREN; CHILDS, SARAH; KANTOLA, JHOANA; KROOK, MONA LENA (2008): «Rethinking Women's Substantive Representation», en: Representation, vol. 44, $\mathrm{n}^{\circ} 2$, pp. 99-110.

CELIS, KAREN; CHILDS, SARAH; KANTOLA, JHOANA; KROOK, MONA LENA (2014): «Constituting Women's Interests through Representative Claims», en: Politics \& Gender, n 10 , pp. 149-174.

CHAPPELL, LOUISE (2002): «The femocrat strategy: Expanding the repertoire of feminist activists», en: Ross, Karen (ed.), Women, Politics and Change, Oxford, Oxford University Press, pp. 85-98.

DAHLERUP, DRUDE (1988): «From a Small to a Large Minority: Women in Scandinavian Politics», en: Scandinavian Political Studies, vol. II, $n^{\circ} 4$, pp. 275-298.

DAHLERUP, DRUDE (2006): «The Story of the Theory of Critical Mass», en: Politics \& Gender, $n^{\circ} 2$, pp. 511-522.

DESPOSATO, SCOTT; NORRANDER, BARBARA (2009): «The Gender Gap in Latin America: Contextual and Individual Influences on Gender and Political Participation», en: British Journal of Political Science, vol. 39, n 1, pp. 141-162.

DODSON, DEBRA (2006): The Impact of Women in Congress, New York, Oxford University Press. FRANCESCHET, SUSAN; PISCOPO, JENNIFER (2008): "Gender Quotas and Women's Substantive Representation: Lessons from Argentina», en: Politics \& Gender, no 4, pp. 393-425.

GREY, SANDRA (2006): «Numbers and beyond: The relevance of critical mass in gender research», en: Politics \& Gender, $\mathrm{n}^{\circ} 2$ 2, pp. 491-530.

HTUN, MALA; JONES, MARK P. (2002): «Engendering the Right to Participate in Decision-Making», en: M. Craske y M. Molyneux (eds.), Gender and the Politics of Rights and Democracy in Latin America, Basingstoke, Palgrave.

JAQUETTE, JANE S. (1997): «Women in Power: From 
Tokenism to Critical Mass», en: Foreign Policy, no 108, pp. 23-37.

KANTER, ROSABETH MOSS (1977): "Some Effects of Proportions on Group Life: Skewed Sex Ratios and Responses to Token Women», en: American Journal of Sociology, vol. 82, n 5, pp. 965-990. KANTOLA, JOHANNA (2006): Feminists Theorize the State, Basingstoke, Palgrave Macmillan.

KANTOLA, JOHANNA; OUTSHOORN, JOYCE (2007): «Changing state feminism», en: Outshoorn, Kantola (ed.) Changing State Feminism, Basingstoke, Palgrave Macmillan, pp. 1-19.

KARP, JEFFREY A. Y BANDUCCI, SUSAN (2008): «When politics is not just a man's game: Women's representation and political engagement», en: Electoral Studies, n² 27, pp. 105-115.

KATHLENE, LYN (1994): «Power and influence in State Legislative Policymaking: The interaction of gender and position in committee hearing debates», en: American Political Science Review, vol. 88, $n^{\circ} 3$, pp. 560-576.

KITTILSON, MIKI CAUL (2005): «In Support of Gender Quotas: Setting New Standards, Bringing Visible Gains», en: Politics \& Gender, vol. 1, $\mathrm{n}^{\circ} 4, \mathrm{pp}$. 638-645.

LAWLESS, JENNIFER L. (2004): «Politics of Presence? Congresswomen and Symbolic Representation Political», en: Research Quarterly, vol. 57, $\mathrm{n}^{\circ}$ 1, pp. 81-99.

LOMBARD0, EMANUELA; MEIER, PETRA (2014): The Symbolic Representation of Gender: a discursive approach, Surrey and Burlington, Ashgate.

LOMBARDO, EMANUELA; MEIER, PETRA (2017): «Capturing in words what a symbol symbolizes? Challenges for studying symbolic representation from a discursive approach», en: Politics, Groups, and Identities, vol. 5, $\mathrm{n}^{\circ}$ 3, 482-487.

LOVENDUSKI, JONI (ED.) (2005): State Feminism and Political Representation, Cambridge/New York,
Cambridge University Press.

MACKAY, FIONA (2008): « Thick> Conceptions of substantive representation: women, gender and political institutions», en: Representation, vol. 44, n² 2, pp. 125-139.

MARX, JUTTA; CAMINOTTI, MARIANA; BORNER, JUTTA (2007): Las legisladoras. Cupos de género y política en Argentina y Brasil, Buenos Aires, Siglo XXI. NORRIS, PIPPA (1996): «Women Politicians: Transforming Westminster?», en: Parliamentary Affairs, vol. 49, nº 1, pp. 89-102.

PÉREZ, VERÓNICA (2014): «Asignación partidaria y especialización: las legisladoras en el sistema de Comisiones de la Cámara de Diputados de Argentina», en: Revista Uruguaya de Ciencia Política, vol. 23, n² 2, pp. 165-184.

PITKIN, HANNA (1967): The concept of representation, Berkeley, University of California Prees.

PIATTI-CROCKER, ADRIANA (2014): "Dos décadas de cuotas de género en Argentina: difusión de políticas y sus efectos", en: N. Archenti y M. I. Tula (comp.), La representación imperfecta. Logros y desafíos de las mujeres políticas, Buenos Aires, Eudeba, pp. 105-128.

RODRÍGUEZ GUSTÁ, ANA LAURA; MADERA, NANCY (2014): "Dimensiones colectivas, relacionales y supranacionales en la construcción de una agenda legislativa de Derechos Humanos de mujeres en América Latina», en: Revista Uruguaya de Ciencias Políticas, vol. 23, pp. 37-64.

SACCHET, TERESA (2008): «Beyond Numbers. The impact of Gender Quotas in Latin America», en: International Feminist Journal of Politics, vol. 10, n 3, pp. 369-386.

SAWARD, MICHAEL (2010): The representative claim, New York, Oxford University Press.

SAWARD, MICHAEL (2012): «Claims and constructions», en: Contemporary Political Theory, vol. $11, n^{\circ} 1$, pp. 123-127. 
SCHWINDT-BAYER, LESLIE A.; MISHLER, WILLIAM (2005): «An Integrated Model of Women's Representation», en: The Journal of Politics, vol. 67, n 2 , pp. 407-428.

STAUDT, KATHLEEN (1996): «Women in politics: Mexico in global perspective», en: Rodríguez V.E.

(ed.), Women's participation in Mexican political life, Boulder, Westview Press.

STOFFEL, SOPHIE (2008): «Rethinking political representation: the case of institutionalized feminist organizations in Chile», en: Representation, vol. 44, no 2, pp. 141-154.

STUDLAR, DONLEY T.; MCALLISTER, IAN (2002): «Electoral systems and women's representation: a long-term perspective», en: Representation, vol. 39, no 1, pp. 3-14.

THOMAS, SUE (1991): «The Impact of Women on State Legislative Policies», en: The Journal of Politics, vol. 53, n 4, pp. 958-976.
THOMAS, SUE (1994): How Women Legislate, New York, Oxford University Press.

WELDON, LAUREN (2002): «Beyond Bodies: Institutional Sources of Representation for Women in Democratic Policymaking», en: The Journal of Politics, vol. 64, no 4, pp. 1153-1174.

WOLBRECHT, CHRISTINA AND CAMPBELL, DAVID E. (2007): "Leading by Example: Female Members of Parliament as Political Role Models», en: American Journal of Political Science, vol. 51, n 4 , October, pp. 921-939.

YODER, JANICE D. (1991): «Rethinking Tokenism. Looking Beyond Numbers», en: Gender \& Society, vol. 5, n², pp. 178-192.

ZETTERBERG, PÄR (2009): «Do Gender Quotas Foster Women's Political Engagement? Lessons from Latin America», en: Political Research Quarterly, vol. 62, no 4 , pp. 715-730. 